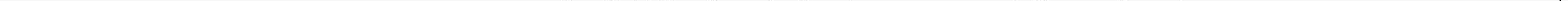




\section{DISCLAIMER}

Portions of this document may be illegible in electronic image products. Images are produced from the best available original document. 


\title{
A Preliminary Systems Assessment of the Starlite Demo Candidates ${ }^{\dagger}$
}

\author{
C. G. Bathke and the ARIES Team \\ - Los Alamos National Laboratory, P.O. Box 1663, Los Alamos, NM 87545, USA
}

\begin{abstract}
The Starlite project has evaluated the following five tokamaks as candidates for the US Demo Power Plant: 1) steady state, first stability regime; 2) pulsed, first stability regime; 3) steady state, second stability regime; 4) steady state, reversed shear; and 5) steady state, low aspect ratio. Systems analysis of these candidates has played an important role in the selection of a reversed-shear tokamak for further conceptual design as a US Demo Power Plant. The cost-based systems analysis that led to the selection of a reversed-shear tokamak is described herein.
\end{abstract}

\section{INTRODUCTION}

The Starlite project has evaluated the following five tokamak physics regimes as candidates to be used in a conceptual design of the US Demo Power Plant: 1) a steady state, first stability regime similar to ARIESI [1]; 2) a pulsed, first stability regime similar to PULSAR [2]; 3) a steady state, second stability regime similar to ARIES-II/IV [3]; 4) a steady state, reversed shear [4]; and 5) a steady state, low aspect ratio $(A)$ [5]. References to these five concepts in this paper are abbreviated as: FS, PU, SS, RS, and LAR, respectively. Systems analysis of these candidates as commercial power plants has played an important role in the selection of only one for detailed conceptual design as the US Demo Power Plant. As part of the systems analysis, cost-optimized designs are generated with the Demo Systems Code (DSC) for each candidate. The tradeoffs that determine the optimal design are unique for each candidate. The mass of the fusion power core (FPC) is minimized in the optimal pulsed design; whereas, minimal FPC mass must be balanced against minimal recirculating power for the optimal steadystate designs. The dominant cost also is affected by the physics regime: magnets dominate the cost of the conventional- $A$ tokamaks $(A=4)$; whereas, the current drive dominates the cost of the LAR design. The RS and SS tokamaks are comparable economically and are the economically most attractive Demo candidates. However, RS is preferred overall for the combination of good economic performance and physics credibility. The details of the comparison of the Demo candidates is presented herein.

TThis work was supported by the U.S. Department of Energy under Contract No. W-7405-ENG-36

\section{MODEL}

A Demo Systems Code (DSC) is being developed that is based on the ARIES Systems Code (ASC) [2,3]. Like the ASC, the DSC examines a spectrum of tokamak reactor options (e.g., different blankets, beta limits, coil scalings, etc.). New DSC capabilities include the addition of reverse shear and low-A tokamak physics regimes and first-of-a-kind costing appropriate for a Demo. The essential features and notation of the DSC models are summarized below.

The axisymmetric plasma is characterized by the major toroidal radius, $R_{T}$; equatorial-plane minor radius, $a_{p}$; vertical elongation, $\kappa$; and triangularity, $\delta$. The plasma beta for the FS is constrained by a Troyon relation: $\beta \equiv 2 \mu_{0}\langle p\rangle / B_{\phi 0}^{2}=C_{T} I_{\phi} / a_{p} B_{T}$; where $\langle p\rangle$ is the volume averaged plasma pressure, $B_{T}$ is the vacuum toroidal magnetic field on axis $\left(R \simeq R_{T}\right.$ ), and the constant $C_{T}$ is limited by ballooning stability. For the SS, the $\beta$ has been reformulated as $\beta \equiv \epsilon\left(\epsilon \beta_{p}\right) S^{2} / q_{*}^{2}$ to illustrate the effects of stability; in this expression $\epsilon \equiv A^{-1}=a_{p} / R_{T}, \beta_{p}$ is the poloidal beta, $S^{2}=\left(1+\kappa^{2}\right) / 2$, and $q_{*}$ is a circularized safety factor. An equilibrium limit constrains $\epsilon \beta_{p} \leq 1.85$, high-n ballooning stability constrains $q_{*} \geq 4.6$, and vertical stability limits $\kappa \leq 2$. Numerical fits to a series of equilibrium and stability calculations are used to determine the normalized beta, $\beta_{N}$, in the scaling $\beta=0.05 \epsilon \beta_{N} S^{2} / q_{*}$ used for PU, RS, and LAR. The preliminary $\beta_{N}$ scalings used in the DSC are shown in Fig. 1 and include a $10 \%$ reduction from stability limits as a disruption safety margin. The FS and PU plasmas represent near-term physics; the PU plasma has a lower $\beta_{N}$ because of profile restrictions imposed by inductive current drive. Advanced physics is represented by SS and RS, and super-advanced physics is represented by LAR.

Central to the DSC is a zero-dimensional, steadystate plasma-power-balance model [6] that includes separate ion and electron energy balance; particle continuity; a specified impurity fraction; charge balance; and a $\beta$ constraint. The zero-dimensional equations are derived from a radial average over plasma profiles that preserve the peak-to-average ratios of pressure and density obtained from detailed equilibrium and current-drive calculations. The fractional fusion power deposited to the ions is calculated from time integrals 


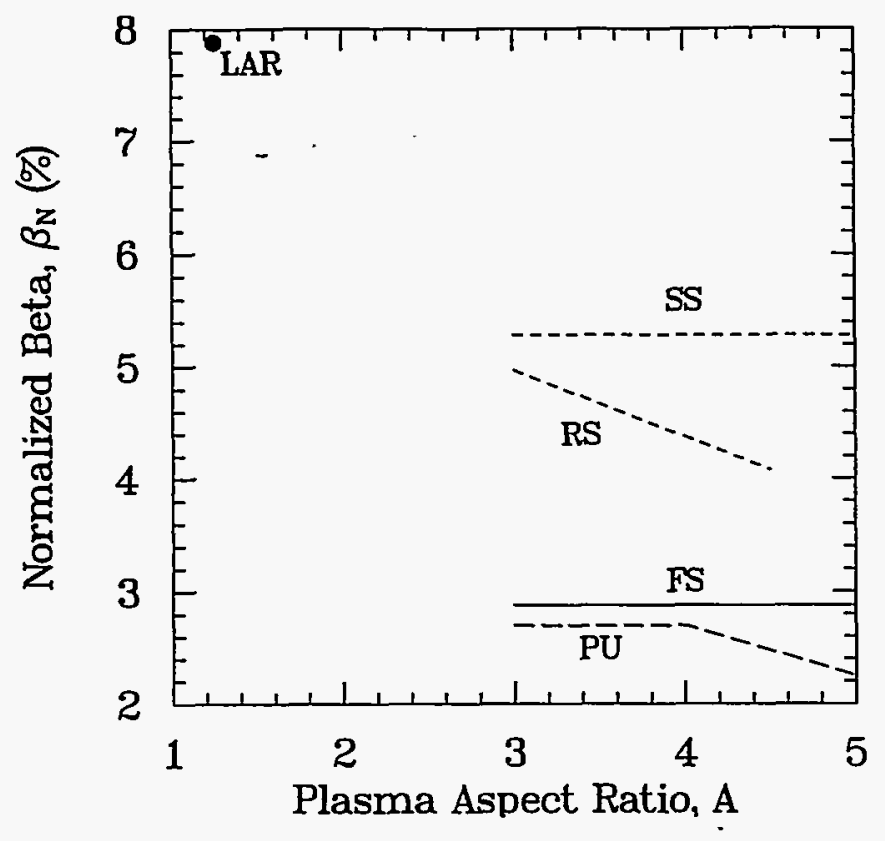

Fig. 1. Scaling of normalized beta with aspect ratio for the five Demo candidates.

of fusion-product slowing down rates. The energy confinement time, $\tau_{E}$, derived from power balance is monitored as the ratio $H=\tau_{E} / \tau_{89 P}$; where $\tau_{89 P}$ is the confinement time predicted by the ITER-89P scaling [7]. The particle confinement time including recycling is assumed to be $\tau_{p}^{*}=10 \tau_{E}$ :

A fraction $f_{B C}$ of the plasma current is sustained by the pressure-gradient-driven bootstrap effect. In both FS and PU, $f_{B C} \propto \epsilon^{1 / 2} \beta_{p}<1$ because of inherently low $\epsilon \beta_{p}$. In SS and LAR $\epsilon \beta_{p}$ is sufficiently large and in RS the pressure gradient is sufficiently large that $f_{B C}$ can exceed unity. Even if $f_{B C} \sim 1$ globally, externally driven current is necessary to match the total driven radial current-density profile with the required equilibrium current-density profile. The location of any mismatch between these two profiles determines the If frequency and power required to drive the needed current. Detailed equilibrium and ray-tracing calculations are used to determine the normalized currentdrive efficiency, $\gamma_{B}=n_{e} 10^{-20} R_{T} I_{p} / P_{C D}$, for each design, as is shown in Fig. 2. The FS and SS designs use a combination of ICRF and LHRF to drive current not sustained by the bootstrap effect. The SS $\gamma_{B}$ is higher than for FS because a greater fraction of the FS driven current is off axis and requires the less efficient LHRF. The SS $\gamma_{B}$ also displays a peak in temperature, because the radial location and type of the current drive shifts from the more efficient on-axis ICRF to the less efficient off-axis LHRF. The RS $\gamma_{B}$ displays a strong sensitivity to changes in $A$, because decreasing $A$ requires more inefficient off-axis current drive. Low field is responsible for the poor current-drive efficiency for

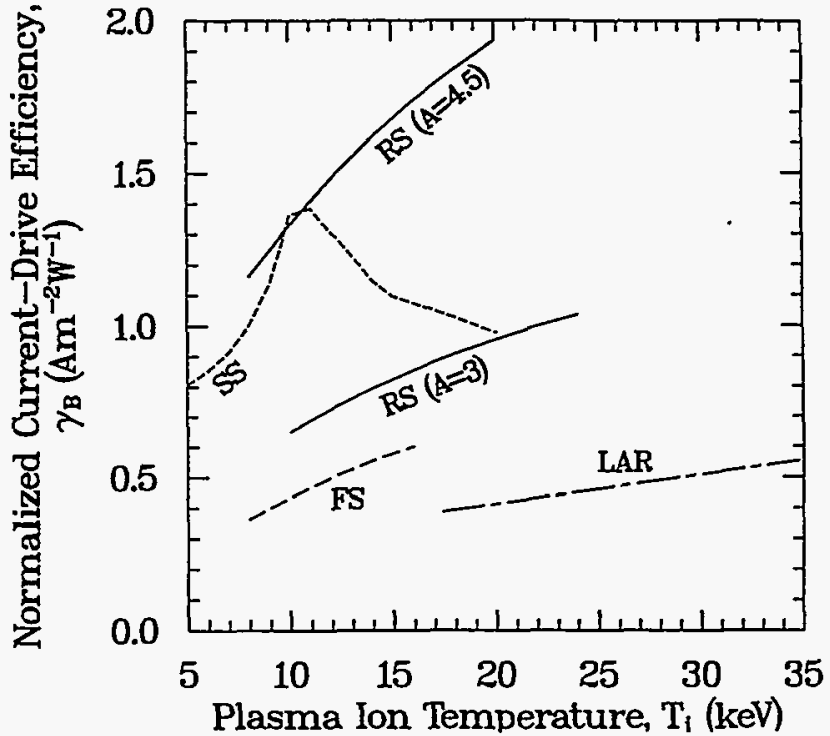

Fig. 2. Scaling of normalized current-drive efficiency with temperature for the five Demo candidates.

LAR. High-frequency, fast wave (HFFW) current drive at approximately the twentieth ion cyclotron harmonic is used for both RS and LAR: $500-900 \mathrm{MHz}$ and 250 MHZ, respectively. The PU design on the other hand inductively drives current by using the PF coils as a transformer.

To compare the five Demo candidates on a purely physics basis, all Demo candidates use the same blanket and shield. The structural material is V5Cr5Ti, and liquid- $\mathrm{Li}$ is both tritium breeder and coolant. The V5Cr5Ti blanket and shield design is similar to that used for ARIES-II [3] and was chosen for its highneutron-wall-loading capability. As is shown in Fig. 3, the combined blanket and shield thickness is $\sim 1.0 \mathrm{~m}$ inboard and $1.4 \mathrm{~m}$ outboard. The PU outboard shield is also used to provide $200 \mathrm{~s}$ of inexpensive ( $\sim 30 \mathrm{M} \$$, or $\sim 80 \$ / M J)$ thermal storage that is used to maintain constant electrical output between plasma-burn periods; additional thermal storage beyond $200 \mathrm{~s}$ is charged at $3.7 \mathrm{M} \$ / \mathrm{s}$ (or $\sim 2 \mathrm{k} \$ / \mathrm{MJ}$ ). A consequence of using the shield for thermal storage is a $3 \%$ reduction in blanket energy multiplication for PU. A gap between the shield and TF coil on the outboard side is provided (for comparative purposes) in all designs to allow blanket sectors to be removed horizontally between TF coils. A scrapeoff thickness of $0.10 \mathrm{~m}$ is used in all designs, except in SS, which has greater compression of adjacent flux surfaces at the equatorial plane relative to the field null and can use $0.05 \mathrm{~m}$.

Constant-tension, D-shaped TF coils are used in all designs but the LAR. The LAR uses a $\mathrm{Cu}$ normal conductor conforming to the shape of the shield, but radially offset from the shield; the center post is flared 


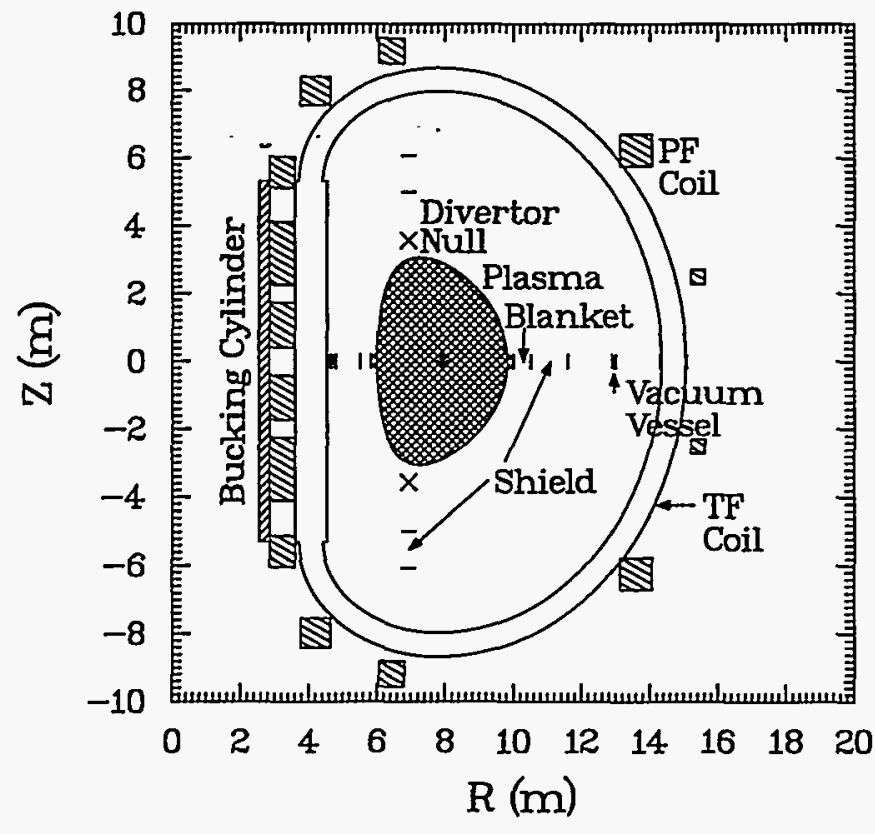

Fig. 3. An elevation view of the pulsed Demo candidate showing the TF and PF coils, the plasma, the bucking cylinder, and the inboard, top/bottom, and outboard radial builds of the first wall, blanket, shield, and vacuum vessel.

to conform to the shape of the plasma (the ratio of average to equatorial-plane cross sectional areas is $\sim 5$ ) and is protected by $0.3 \mathrm{~m}$ of shield to ensure Class $\mathrm{C}$ waste disposal for the center post. Even then the center post must be changed out yearly to prevent the build up of transmutations products that increase resistivity. For the reamining designs, the superconductor is $\mathrm{Nb}_{3} \mathrm{Sn}$, and is limited to $\leq 16 \mathrm{~T}$. Cyclic-fatigue effects require the engineering current density, $j_{T F}$, be decreased for pulsed operation as the number of pulses are increased to ensure survival of the TF coils for the plant lifetime. For PU the TF-coil bucking forces are transmitted through the inboard PF-coil stack to the bucking cylinder; whereas the bucking cylinder is positioned between the TF and PF coils for the conventional- $A$, steady-state designs. The LAR has no bucking cylinder.

A PF-coil model that was developed for PULSAR [2] and is shown in Fig. 1 is used for PU, RS, and LAR; the ARIES PF-coil model [3] that lumps the PF-coils into divertor and equilibrium-field coil pairs is used for FS and SS. The PULSAR model generates a PFcoil set with minimum stored energy that produces the required equilibrium field at full and partial beta with full plasma current and the magnetic flux swing required to: induce and sustain the plasma current for pulsed plasmas and induce the plasma current for steady-state, conventional- $A$ plasmas. No fluxswing requirement is imposed for LAR. The model also

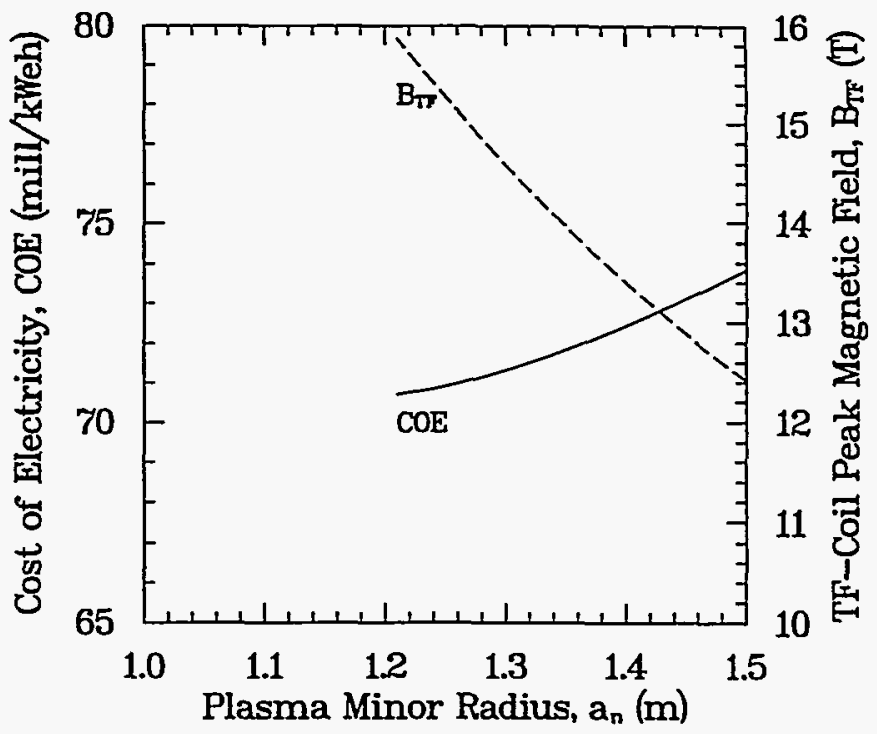

Fig. 4. Variation of the cost of electricity and peak field at the TF-coil with plasma minor radius.

determines the stress in the keys positioned between inboard (straight) legs of pulsed TF coils to react the TF-coil overturning forces. A simplified circuit model is used to determine the power-supply requirements (i.e., reactive power) for pulsed operation.

The DSC costing algorithm allows for a detailed cost breakdown to four levels. The cost scalings used are consistent with U.S. fission nuclear-reactor experience [8]. Credits for appropriate Levels of Safety Assurance (LSA) [9] are included. Costing capabilities for both commercial and Demo power plants [10] are provided in the DSC.

\section{RESULTS}

Parameters that are generated with DSC for costoptimized, commercial power plants are given in Table I. Although the COE optimum aspect ratio is $A \sim 3.5$, the aspect ratio has been limited to $A=4$ for FS, PU, and SS to improve the layout, accessibility, and maintainability of the FPC with little impact on the COE; exceptions are RS and LAR. Preliminary aspect ratios for these two have been taken from [4] and [5], respectively. All designs have been optimized in minor radius. The three all-superconducting, steadystate designs (FS, RS, and SS) optimize the COE at the minimum minor radius at which the peak field at the TF coil is $16 \mathrm{~T}$, as is illustrated in Fig. 4 for RS. The PU design optimizes the COE at the minor radius that maximizes mass power density, MPD; at smaller radii MPD decreases because the TF-coil mass increases, and at larger radii MPD decreases because the shield volume increases. The optimum plasma temperature is generally a trade off between increasing $\gamma_{B}$ and decreasing fusion power density 
TABLE I Parameters for the Five Demo Candidates.

\begin{tabular}{|c|c|c|c|c|c|c|}
\hline Parameter - . & FS & PU & $\mathrm{RS}$ & SS & LAR & \\
\hline Length of burn, $\tau_{B}(\mathrm{~h})$ & $\infty$ & 2.5 & $\infty$ & $\infty$ & $\infty$ & \\
\hline Plasma aspect ratio, $A=R_{T} / a_{p}$ & 4.0 & 4.0 & 4.5 & 4.0 & 1.25 & \\
\hline Major toroidal radius, $R_{T}(\mathrm{~m})$ & 7.80 & 7.92 & 5.45 & 5.76 & 5.59 & \\
\hline Plasma minor radius, $a_{p}(\mathrm{~m})$ & 1.95 & 1.98 & 1.21 & 1.44 & 4.47 & \\
\hline Plasma elongation, $\kappa_{95}$ & 1.61 & 1.60 & 1.80 & 1.80 & 2.20 & \\
\hline Plasma elongation, $\kappa_{x}$ & 1.81 & 1.80 & 2.00 & 2.03 & 2.40 & \\
\hline Plasma triangularity, $\delta_{95}$ & 0.47 & 0.35 & 0.50 & 0.48 & 0.50 & \\
\hline Plasma triangularity, $\delta_{x}$ & 0.71 & 0.50 & 0.75 & 0.67 & 0.60 & \\
\hline Circularized safety factor, $q_{*}$ & 3.77 & 2.40 & 2.37 & 4.60 & 2.38 & \\
\hline Stability parameter, $\epsilon \beta_{p}$ & 0.54 & 0.32 & 0.48 & 1.22 & 0.94 & \\
\hline Normalized beta, $\beta_{N}(\%)$ & 2.88 & 2.70 & 4.08 & 5.28 & 7.88 & \\
\hline Toroidal beta, $\beta(\%)$ & 2.03 & 2.50 & 4.06 & 3.04 & 38.6 & \\
\hline Poloidal beta, $\beta_{p}$ & 2.17 & 1.30 & 2.17 & 4.86 & 1.17 & \\
\hline Ion temperature, $T_{i}(\mathrm{keV})$ & 14.0 & 14.0 & 14.0 & 10.0 & 17.4 & \\
\hline Electron temperature, $T_{e}(\mathrm{keV})$ & 15.0 & 14.2 & 15.0 & 10.3 & 19.0 & \\
\hline Ion density, $n_{i}\left(10^{20} / \mathrm{m}^{3}\right)$ & 1.15 & 1.15 & 2.01 & 2.07 & 0.68 & \\
\hline Electron density, $n_{e}\left(10^{20} / \mathrm{m}^{3}\right)$ & 1.35 & 1.46 & 2.42 & 2.41 & 0.81 & \\
\hline Confinement ratio, $\tau_{p}^{*} / \tau_{E}$ & 10.0 & 10.0 & 10.0 & 10.0 & 10.0 & \\
\hline Lawson parameter, $n_{i} \tau_{E}\left(10^{20} \mathrm{~s} / \mathrm{m}^{3}\right)$ & 1.71 & 4.13 & 2.36 & 2.69 & 1.76 & \\
\hline Ignition parameter, $\beta \tau_{E} / a_{p}^{2}\left(\% \mathrm{~s} / \mathrm{m}^{2}\right)$ & 0.79 & 2.29 & 3.26 & 1.91 & 5.03 & - \\
\hline ITER-89P scaling [7] multiplier, $H$ & 1.72 & 2.36 & 2.13 & 2.89 & 2.37 & \\
\hline Normalized confinement multiplier, $H / q_{95}$ & 0.38 & 0.67 & 0.61 & 0.24 & 0.13 & \\
\hline Radiation fraction, $f_{R A D}$ & 0.16 & 0.31 & 0.18 & 0.18 & 0.11 & \\
\hline Plasma current, $I_{p}(\mathrm{MA})$ & 12.5 & 14.7 & 10.0 & 6.84 & 38.8 & \\
\hline Bootstrap-current fraction, $f_{B C}$ & 0.57 & 0.34 & 0.84 & 0.87 & 0.85 & \\
\hline $\mathrm{CD}$ efficiency, $\gamma_{B}\left(10^{20} \mathrm{~A} / \mathrm{W} \mathrm{m}^{2}\right)$ & 0.56 & $\mathrm{NA}$ & 1.62 & 1.36 & 0.41 & \\
\hline CD power to plasma, $P_{C D}(\mathrm{MW})$ & 236.3 & 0 & 81.7 & 69.9 & 432.0 & \\
\hline On-axis toroidal field, $B_{T}(\mathrm{~T})$ & 9.06 & 8.00 & 8.31 & 8.25 & 1.77 & \\
\hline Peak field at TF coil, $B_{T F}(\mathrm{~T})$ & 15.9 & 13.9 & 15.9 & 15.9 & 14.8 & \\
\hline TF-coil current density, $j_{T F}\left(\mathrm{MA} / \mathrm{m}^{2}\right)$ & 26.5 & 15.0 & 32.7 & 31.3 & $35-6$ & \\
\hline TF-coil ohmic losses, $P_{T F}(\mathrm{MW})$ & NA & $\mathrm{NA}$ & $\mathrm{NA}$ & $\mathrm{NA}$ & 773.2 & \\
\hline Peak neutron load, $\hat{I}_{w}\left(\mathrm{MW} / \mathrm{m}^{2}\right)$ & 2.68 & 2.18 & 5.37 & 4.87 & 3.88 & \\
\hline First-wall/blanket life, $I_{w} \tau\left(\mathrm{MW} \mathrm{yr} / \mathrm{m}^{2}\right)$ & 16.4 & 16.4 & 16.4 & 16.4 & 16.4 & \\
\hline Normalized heat flux, $P_{T R} / R_{T}(\mathrm{MW} / \mathrm{m})$ & 71.8 & 32.2 & 68.3 & 63.4 & 201.6 & \\
\hline Blanket energy multiplication, $M_{N}$ & 1.39 & 1.35 & 1.39 & 1.39 & 1.39 & \\
\hline Thermal conversion efficiency, $\eta_{T H}$ & 0.46 & 0.46 & 0.46 & 0.46 & 0.46 & \\
\hline Recirculating power fraction, $\epsilon$ & 0.29 & 0.06 & 0.14 & 0.17 & 0.63 & \\
\hline Mass power density, MPD (kWe/tonne) & 44.0 & 29.6 & 86.2 & 73.6 & 15.1 & \\
\hline Level of Safety Assurance, LSA & 2 & 2 & 2 & 2 & 2 & \\
\hline Dollar year & 1992 & $199 \overline{2}$ & 1992 & 1992 & 1992 & \\
\hline Total direct cost, TDC (B\$) & 2.74 & 3.46 & 2.06 & 1.98 & 3.65 & \\
\hline Total capital cost, TCC (B\$) & 5.30 & 6.68 & 3.97 & 3.82 & 7.04 & \\
\hline \multicolumn{7}{|l|}{ Cost of electricity (mill/kWeh): } \\
\hline total COE & 89.72 & 108.84 & 70.70 & 68.56 & 116.55 & \\
\hline total COE w/o safety credits ( $\mathrm{LSA}=4)$ & 101.87 & 123.40 & 80.57 & 78.13 & 131.72 & \\
\hline
\end{tabular}




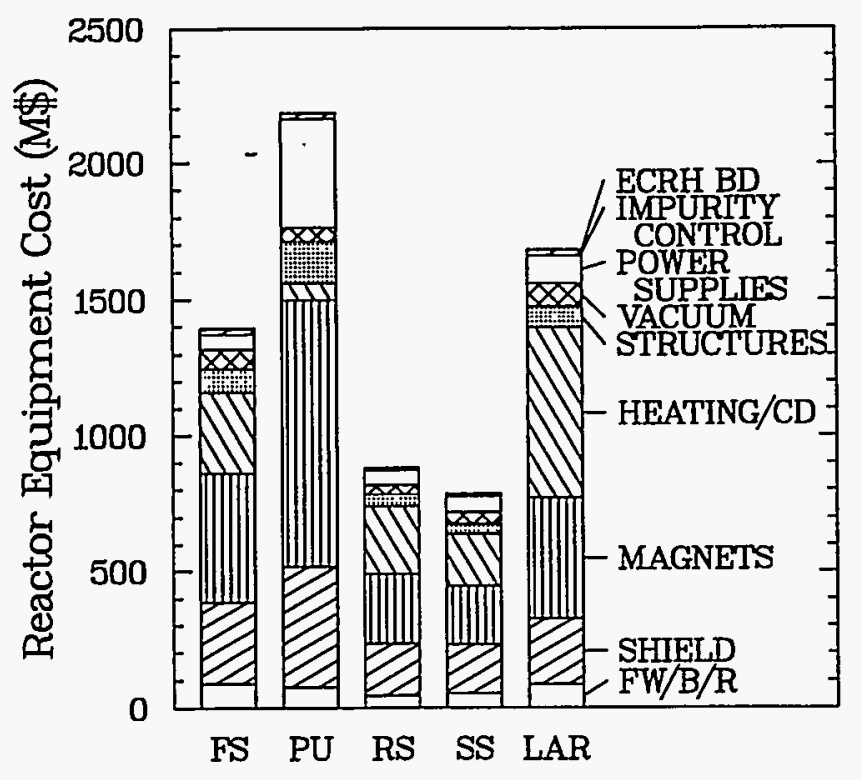

Fig. 5. Reactor Equipment Costs for the five Demo candidates.

with increasing temperature. For SS the optimum plasma temperature is determined solely by the peak in $\gamma_{B}$ (see Fig. 2).

The Reactor Equipment Costs, which in total are equivalent to the FPC cost, are given in Fig. 5 for the five Demo candidates. The PU design has the largest FPC cost, which is dominated by magnet and power-supply costs. The high magnet cost results from extra structure required to fortify against cyclic-fatigue effects, and the power-supply cost is high relative to steady-state operation because of inductive currentdrive requirements. In spite of a larger $\beta_{N}$ and $\beta$, LAR has a larger FPC cost relative to RS and SS. The larger plasma current and poorer current-drive efficiency make the cost of the current-drive system so expensive. Also, extra conductor is added to the normal conducting TF coil to reduce ohmic dissipation and recirculating power. Because of a lower $\beta$, FS has a more expensive FPC than either RS or SS. The FPC costs of RS and SS are comparable because the geometrical (i.e., $A), \beta$, and current-drive differences cancel each other economically. Compiling the Total Direct Costs (TDC) for the five Demo candidates indicates that LAR is further penalized for a large recirculating power with a relatively larger turbine-plant-equipment cost, as is shown in Fig. 6.

The COE for each Demo candidate is given in Fig. 7 . The Starlite requirement ( $\leq 80 \mathrm{mill} / \mathrm{kWeh}$ ) and goal $(\leq 65 \mathrm{mill} / \mathrm{kWeh}$ ) for the COE $[10,11]$ of a commercial power plant are also indicated in Fig. 7. Neither LAR, PU, nor FS meet the COE requirement; LAR fails

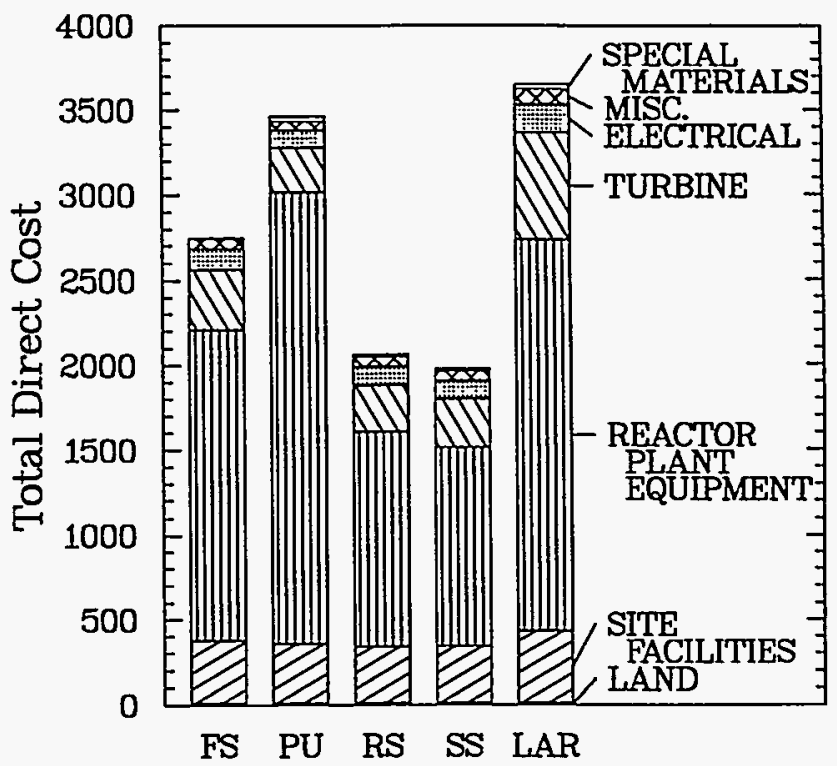

Fig. 6. Total Direct Costs for the five Demo candidates.

because of its large recirculating power and associated costs, PU fails because of its low $\beta$ and MPD and cost penalties associated with pulsed operation, and FS fails because of its low $\beta$ and MPD. The COE requirement is meet by RS and SS because of high $\beta$ and MPD and low current-drive and recirculating power. Economically, RS and SS are comparable and the most attractive of the five Demo candidates. No Demo candidate surpasses the COE goal.

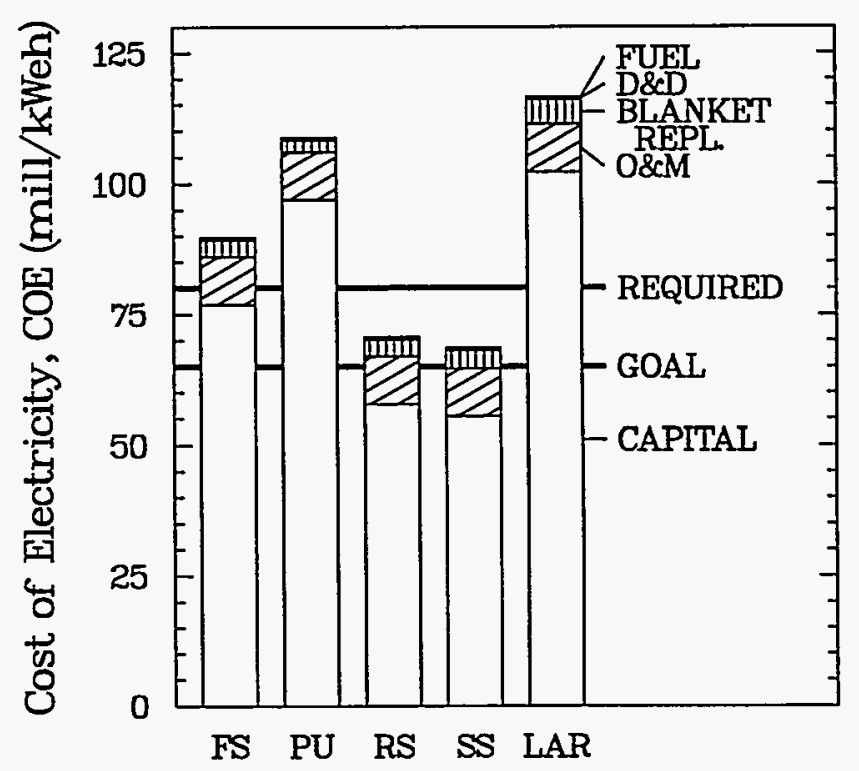

Fig. 7. Cost of Electricity for the five Demo candidates. The Starlite COE requirement and goal for a commercial power plant are also indicated. 


\section{SUMMARY}

The Starlite project has generated cost-optimized design parameters for commercial power plants with common engineering features that are based on the following five tokamak physics regimes: 1) a steady state, first stability regime (FS) similar to ARIES-I [1]; 2) a pulsed, first stability regime (PU) similar to PULSAR [2]; 3) a steady state, second stability regime (SS) similar to ARIES-II/TV [3]; 4) a steady state, reversed shear tokamak (RS); and 5) a steady state, low aspect ratio tokamak (LAR). Preliminary systems analyses indicate that only the RS and SS meet the Starlite requirement on COE for a commercial power plant. However, the RS was judged overall to be the most attractive commercial power plant because of its economic performance and relative physics credibility $[11,12]$. For this reason, the Starlite project is undertaking a conceptual design of the US Demo Power Plant based on reversed-shear tokamak physics.

\section{REFERENCES}

[1] F. Najmabadi, et al., "The ARIES-I Tokamak Reactor Study," University of California Los Angeles report UCLA-PPG-1323 (1991).

[2] F. Najmabadi, et al., "The PULSAR Tokamak Reactor Study," University of California San Diego report (to be published).

[3] F. Najmabadi, et al., "The ARIES-II and -IV Second Stability Tokamak Reactors," University of California Los Angeles report UCLA-PPG-1461 (to be published).

[4] C. Kessel, J. Manickam, G. Rewoldt, and W. M. Tang, "Improved Plasma Performance in Tokamaks with Negative Magnetic Shear," Phys. Rev. Lett. 72 8 (February 1994) 1212.

[5] Y-K M. Peng, "Prospects and Status of Low-AspectRatio Tokamaks," Trans. Fus. Technol. 27 (April 1995) 138.

[6] K A. Werley, "Reversed Field Pinch Ignition Requirements," Nuclear Fusion 31 (1991) 576.

[7] D. E. Post, K Borrass, J. D. Callen, S. A. Cohen, J. G. Cordey, F. Engelmann, et al., "ITER Physics," International Thermonuclear Experimental Reactor documentation series No. 21 (November 1990).

[8] J. G. Delene et al., "Nuclear Energy Cost Data Base," US DOE report DOE/NE-0095 (1988).
[9] J. P. Holdren et al., "Report of the Senior Committee on Environmental, Safety, and Economic Prospects of Fusion Reactors," Lawrence Livermore National Laboratory report UCRL-53766 (1989).

[10] R. L. Miller and the ARIES Research Team, "Starlite Economics: Requirements and Methods," this conference.

[11] F. Najmabadi, L. Waganer, and the ARIES Research Team, "The Starlite Project: The Mission of the Fusion Demo and Preliminary Assessment of Demo Candidates," this conference.

[12] T. K Mau, D. A. Ehst, S. C. Jardin, C. E. Kessel, B. J. Lee, and the ARIES Research Team, "Plasma System Requirements and Performance Data Base for the Starlite/Demo Fusion Power Plant," this conference. 